Bibliotecas. Vol 40, № 1, enero - junio, 2022. EISSN: 1659-3286

URL: http://www.revistas.una.ac.cr/index.php/bibliotecas/index

DOI: http://dx.doi.org/10.15359/rb.40-1.5

Licencia: Creative Commons (BY-NC-SA) 4.0 Internacional

\title{
Una experiencia educativa en el área de la bibliotecología: innovando por medio de estrategias didácticas prácticas y creativas
}

An Educational Experience in the Area of Librarianship: Innovating Through Practical and Creative Teaching Strategies

Lorena Chaves Salgado

Universidad de Costa Rica, Costa Rica

ORCID ID: https://orcid.org/0000-0001-9169-7541

Daniela Rodríguez Valerio

Universidad de Costa Rica, Costa Rica

ORCID ID: https://orcid.org/0000-0003-3737-8851

Ericka Méndez Chacón

Universidad de Costa Rica, Costa Rica

ORCID ID: https://orcid.org/0000-0003-3152-5179

Recibido:10 de febrero de 2021

Aceptado:05 de agosto de 2021

Publicado: 25 de enero de 2022

\begin{abstract}
Resumen:
El artículo tiene como objetivo presentar el proceso desarrollado para adecuar, innovar y mejorar las estrategias didácticas aplicadas en el curso "Fundamentos de las Ciencias Bibliotecológicas y de la Información" de la Escuela de Bibliotecología y Ciencias de la Información de la Universidad de Costa Rica. Para estos efectos se realizó un diagnóstico a 56 estudiantes matriculados durante el I ciclo 2019 y con base en sus necesidades y formas de aprender se idearon y aplicaron 3 estrategias didácticas que facilitaron la obtención de conocimientos, un aprendizaje significativo y la adquisición de habilidades blandas. Como resultado, las actividades desarrolladas tuvieron una gran aceptación por parte de los estudiantes, lo cual se vio reflejado tanto en sus evaluaciones como en las aplicadas a las docentes.
\end{abstract}

Palabras clave: Bibliotecología; Didáctica; Aprendizaje significativo; Educación; Docencia.

\begin{abstract}
:
The article aims to present the process developed to adapt, innovate and improve the teaching strategies applied in the course "Fundamentals of Library and Information Sciences" of the School of Library and Information Sciences of the University of Costa Rica. For this purpose, a diagnosis was made to 56 students enrolled during the I cycle 2019 and based on their needs and ways of learning, 3 didactic strategies that facilitated the acquisition of knowledge, meaningful learning and the acquisition of soft skills were devised and applied. As a result, the activities carried out were widely accepted by the students, which was reflected both in their evaluations and those applied to teachers.
\end{abstract}

Keywords: Librarianship; Didactics; Meaningful Learning; Education; Teaching. 
"Dime y lo olvido, enséñame y lo recuerdo, involúcrame y lo aprendo" Benjamín Franklin

\section{Introducción}

En Costa Rica, la carrera de bibliotecología se imparte desde el año 1968, siendo la Universidad de Costa Rica la institución que comenzó con la enseñanza de esta disciplina. Desde entonces a la fecha la carrera se ha caracterizado por permanecer en constante evolución y marcada por cambios concretos en el proceso de cómo se aprende, lo que ha dado paso al desarrollo de diferentes formas de enseñanza adecuadas a las demandas de la población estudiantil. Lo anterior ha implicado para los docentes dejar atrás muchas de las formas de enseñanza tradicionales para dar paso a planteamientos más novedosos, creativos y efectivos que ayuden al estudiante a desarrollarse con un rol más activo al involucrarse en prácticas docentes más significativas.

En el caso particular de los programas de estudio del bachillerato de la Escuela de Bibliotecología y Ciencias de la Información de la Universidad de Costa Rica se ofrecen actualmente dos énfasis: Ciencias de la Información y Bibliotecas Educativas. La gran diferencia entre ambos es que el segundo está orientado a formar profesionales que se desempeñarán en su mayoría en el ámbito educativo. Ambos bachilleratos cuentan con un tronco común que abarca 3 años de estudio y es en el cuarto año donde se cursa la especialidad.

Cabe recalcar que para la presente investigación se trabajó durante 4 meses con 56 estudiantes del curso Bl-1002 Fundamentos de las Ciencias Bibliotecológicas y de la Información, se investigaron las características propias de la población en estudio y de acuerdo con esta información obtenida se diseñaron y aplicaron estrategias didácticas significativas y específicas: un taller, una gira educativa y una clase teórica acompañada de un trabajo práctico manual en equipo, con el fin de determinar el impacto en el aprendizaje y motivación de los estudiantes respecto a los contenidos del curso. 


\section{Referentes teóricos}

\section{a. El ser humano: un individuo que aprende}

Se reconoce que el ser humano se comunica, es social y mediante la interacción con su entorno aprende. Es la vivencia y el sometimiento a experiencias lo que estimula la adopción de aprendizajes y el desarrollo de competencias.

En este proceso, es importante distinguir que los individuos se exponen a hechos educativos y hechos pedagógicos. González (2009) explica que el hecho educativo se refiere a aquellas vivencias que no se planifican, no son intencionales y en las que no media una intención formativa, el hecho educativo es una situación evolutiva que también comparten los animales y que les permite a los seres aprender y adaptarse al entorno (citado en Piedra y Arias, 2015).

Por otra parte, está el hecho pedagógico, el cual se entiende, según Suescún y Wilberth (2012), como aquellos procesos planificados, intencionados y gestionados para estimular la formación de una persona (citado en Piedra y Arias, 2015). El hecho pedagógico es mediado por un docente y en él se involucran procesos de comunicación, interacción y emocionales. Piedra y Arias (2015) explican además que en este hecho pedagógico influyen también las creencias y representaciones de los propios estudiantes, su desempeño y la actitud de los docentes; aclaran que el proceso de enseñanza va más allá de impartir contenidos, sino que convergen procesos cognitivos, sociales y emotivos.

Es importante destacar el papel del involucramiento entre seres humanos para estimular el aprendizaje, ya que "el conocimiento no es un objeto que se pasa de uno a otro, sino que es algo que se construye por medio de operaciones o habilidades cognoscitivas que se inducen en la interacción social" (Mejías y Sandoval, 1996, p. 38 citado en González y León, 2009, p. 30). Los autores recalcan, además, que este es un proceso que demanda capacidades intelectuales, conceptuales, lingüísticas e informativas.

\section{b. El acto educativo mediado a través de actividades prácticas y la interacción entre individuos}

A través de los años se ha evidenciado la necesidad de involucrar a los estudiantes en procesos de enseñanza que estimulen la socialización y posibiliten un rol más activo por 
parte de estos, debido a esta realidad es relevante implementar estrategias didácticas con actividades prácticas y lúdicas, donde lo lúdico se refiere a generar en el aula espacios más creativos que enriquecen los procesos de enseñanza aprendizaje por medio del planteamiento y ejecución de nuevos enfoques de conocimiento dirigidos a los estudiantes, que junto con el profesor desarrollan propuestas de participación que educan para la vida (Araya, 2016).

"El reconocimiento de la lúdica, como condición intrínseca y esencial (no exclusivo) de la naturaleza humana, es, por lo tanto, fundamental para quien estudia los procesos de aprendizaje del ser humano, pues esta condición traspasa todas las funciones del "ser", y una función elemental de la vida es "aprender"' (Araya, 2016, p.21)

Las propuestas educativas mediadas por lo lúdico deben construirse en un clima de confianza, jugar con el ritmo del fluir, buscar otros espacios diferentes al aula y fortalecer el aprendizaje dirigido hacia enfoques pedagógicos que desarrollen los saberes por medio de propuestas teóricas-conceptuales combinadas con trabajo práctico que incremente el aprendizaje del estudiante. Para efectos de este artículo, se construyeron tres estrategias didácticas que toman conceptos de lo lúdico, por ejemplo, se fomentaron la convivencia, las experiencias, el intercambio de ideas y se dio espacio para desarrollar la creatividad por medio de las evaluaciones asignadas.

\section{c. Consideraciones para el trabajo con grupos grandes en el ámbito universitario}

Al escoger una estrategia didáctica, debemos considerar varios aspectos, entre ellos: los objetivos de lo que queremos transmitir, el tamaño y la madurez del grupo, el ambiente o el contexto de la clase, el tiempo con el que contamos, las características de los alumnos, así como la personalidad del educador, asimismo, se debe hacer una valoración de las ventajas e inconvenientes que podría acarrear la estrategia (Imbernon y Medina, 2006).

En este caso, un aspecto muy importante es el tamaño del grupo. Se considera que un grupo grande es aquel de cincuenta personas o más, sin embargo, este es un criterio de cardinalidad. Para definir un grupo grande median además las estrategias didácticas utilizadas por el profesor (Piedra García, 2015). 
El tamaño del grupo incide en el aprendizaje, Sobrados (2016) comenta que en grupos grandes el profesor tiene que realizar un esfuerzo para mantener la disciplina y la atención, además de complementar la enseñanza tradicional con metodologías y recursos que consigan el desarrollo de competencias. Explica, además, que bajo esta situación se tiende a "masificar", lo cual reduce la calidad de la enseñanza.

Un grupo grande, por ejemplo, podría experimentar una interacción más limitada y el rol del profesor, sus estrategias didácticas y modelos de evaluación cambian. La existencia de grupos grandes en espacios educativos universitarios ha sido un tema con gran interés y esta realidad se explica por varias razones, entre ellas el aumento de la población estudiantil, las limitaciones presupuestarias y de espacio, políticas administrativas de la universidad, estudiantes en cursos de servicio e incluso por tradiciones de enseñanza (Piedra García, 2015).

Es importante recalcar que en el trabajo con grupos grandes la cooperación heterotécnica ha mostrado "generar y organizar el conocimiento de forma más compleja, superando relaciones de causa-efecto simples y creando diversos puntos de vista y opiniones sobre un mismo tema" (Piedra García, 2015, p. 25). El autor explica que el ser humano es un ser social y que aprende interactuando, por lo tanto, considerar estrategias didácticas en las que los individuos socialicen y tengan que coordinar entre síse consideran buenas prácticas para incentivar el aprendizaje de grupos grandes en el contexto universitario. Se destaca además que la cooperación heterotécnica puede promoverse mediante el trabajo en equipo, lo cual le brinda al docente la posibilidad de brindar una atención más personalizada a los estudiantes.

\section{d. El caso del curso "Fundamentos de las Ciencias Bibliotecológicas y de la información de la Universidad de Costa Rica"}

Este es un curso impartido como parte del plan de estudios de las carreras de Bachillerato en Bibliotecología con Énfasis en Ciencias de la Información y el Bachillerato en Bibliotecología con énfasis en Bibliotecas Educativas, ambas ofertadas por la Universidad de Costa Rica. De acuerdo con la Escuela de Bibliotecología y Ciencias de la Información (EBCl, 2019) este es un curso que busca brindar al estudiante un panorama amplio acerca de la carrera y su impacto en la sociedad a través del tiempo. Entre sus contenidos destacan la terminología especializada en el área, la historia de la escritura, las bibliotecas, 
el libro y los tipos de bibliotecas que se encuentran en el país (nacional, pública, escolar, municipal, universitaria, especializada y el Learning Commons del Instituto Tecnológico de Costa Rica).

Es importante mencionar que los Learning Commons son espacios para el trabajo colaborativo en bibliotecas académicas, los cuales han surgido a raíz de la gradual desaparición del libro impreso, el aumento en el acceso a documentos digitales y una creciente demanda de entornos sociales para el trabajo académico y colaborativo, entre otros (González, 2013).

En el curso también se estudia la historia de la carrera de bibliotecología, además el rol social del bibliotecólogo enmarcado en la Agenda 2030 de Naciones Unidas que promueve el desarrollo sostenible y el campo de trabajo y académico del profesional en esta área. Un elemento para destacar es que es un curso impartido a estudiantes de primer año de forma presencial y que demanda al estudiante un total de doce horas de trabajo en clase y extra clase. Es, además, de tipo teórico y usualmente se ofrece en grupos grandes, es de relevancia, pues a los estudiantes les aclara en qué consiste la carrera. Este conocimiento adquirido muchas veces influye en su decisión de abandonarla o permanecer en ella, dado que en Costa Rica la bibliotecología se sigue percibiendo, en algunos ámbitos, desde su enfoque tradicional.

\section{Metodología}

La presente investigación se realizó por medio de un alcance exploratorio el cual, de acuerdo con Muñoz Rocha (2015), se utiliza para comenzar a indagar sobre un tema y no es profundo.El enfoque es cuantitativo, pues la recolección de datos se fundamenta en la medición, se presentan mediante números (en su mayoría) y se analizan a través de métodos estadísticos, su interpretación constituye una explicación de cómo los resultados encajan con el conocimiento existente y se busca generalizar (Hernández, Fernández y Baptista, 2010).

Este trabajo nació con el objetivo de adecuar, innovar y mejorar las estrategias didácticas pensadas inicialmente para el curso "Fundamentos de las Ciencias Bibliotecológicas y de la Información", un curso teórico que se imparte a nivel de Bachillerato y en el primer año de las carreras de Bibliotecología de la Universidad de Costa Rica, cuando los estudiantes 
están viviendo un proceso de transición a la educación superior y de acuerdo con el Programa Estado de la Educación (2019) usualmente vienen de trabajar bajo modelos de enseñanza tradicionales, lo cual se buscó evitar en este curso introductorio.

Se trabajó con 56 estudiantes durante 4 meses, a partir del 28 de marzo de 2019 y hasta el 27 de junio de 2019. Esta iniciativa se emprendió con miras a desarrollar en el aula un aprendizaje más significativo y alejado de la mirada de la educación tradicional, considerando que las carreras se encuentran en proceso de reacreditación como parte de los objetivos de mejora continua de la Universidad.

Para iniciar esta investigación se redactó un consentimiento informado que se presentó a los estudiantes para su aprobación en la segunda clase del curso. Por medio de este consentimiento se les explicó de forma escrita y verbal la dinámica de participación de ellos como sujetos de investigación, además se les aplicó un instrumento para diagnosticar y conocer aspectos personales de la vida de los estudiantes que pudieran tener alguna influencia en sus formas de aprender.

Posteriormente se planearon tres intervenciones didácticas considerando las características del grupo: un taller, una gira educativa y una clase magistral acompañada de un trabajo práctico manual y en equipo. Para cada una de las estrategias se elaboró un instrumento de evaluación aplicado a los sujetos de investigación ocho días después de cada actividad, cabe recalcar que estas evaluaciones suministraron la información pertinente para detectar el impacto de las estrategias y cumplir con el objetivo de la investigación.

\section{a. Etapa 1: Evaluación de las características de los sujetos de investigación}

Por medio de un cuestionario se buscó diagnosticar la situación de aprendizaje y caracterizar a los estudiantes del curso: su edad, género, carrera matriculada, nivel de carrera, si tenían beca y si bibliotecología era la carrera que realmente deseaban estudiar. También se indagó, a través de preguntas cerradas, sobre los siguientes aspectos que podrían afectar el proceso de aprendizaje:

- Influencia del entorno físico y el horario del curso.

- Necesidad de desarrollar trabajo individual y/o colaborativo.

- Realizar discusiones en clase. 
- Aprender haciendo.

- Pertinencia de actividades prácticas y creativas dentro y fuera del aula.

- Importancia de disponer de rúbricas de evaluación.

- Influencia del uso de tecnologías en el aprendizaje.

- Relación con el docente.

- Asignación de tiempo de descanso durante el desarrollo de cada clase.

- Trabajo extracurricular y responsabilidad de parte del estudiante.

- Fortalezas personales que ayudan a aprender.

- Actividades que facilitan el aprendizaje.

Referente a preguntas abiertas se investigó sobre lo siguiente:

- Maneras efectivas de aprender.

- Estrategias didácticas sugeridas para el curso.

Se destaca que por medio de estas variables se recolectó información necesaria para adecuar las estrategias didácticas a las necesidades específicas de la población participante en la investigación y, con ello, conseguir un impacto positivo en el aprendizaje de los contenidos del curso.

\section{b. Etapa 2: Planeamiento y ejecución de estrategias didácticas}

Considerando la información obtenida por el cuestionario aplicado para diagnosticar y caracterizar a los estudiantes, se determinó diseñar y poner en práctica tres estrategias didácticas adecuadas al grupo en estudio y a los contenidos del curso, presentadas en la Tabla 1.

\section{c. Etapa 3: Evaluación del impacto de las estrategias didácticas}

Cada estrategia didáctica fue evaluada ocho días después de su aplicación, con el fin de que el grupo de estudiantes tuviera la oportunidad de asimilar el conocimiento adquirido y evaluar sin presión de tiempo. Cabe destacar que en las evaluaciones no participó necesariamente la totalidad del grupo, ya que este fue un proceso voluntario y que, además, se vio afectado por las ausencias a las clases.

Dicha evaluación se realizó mediante un cuestionario adecuado a cada estrategia. Todos los instrumentos contenían el título de la actividad a evaluar, instrucciones para completar los apartados y se solicitó información general de cada estudiante a través de preguntas cerradas (edad, género, carrera que cursa). En la Cuadro 1 se presentan los aspectos evaluados. 


\section{Cuadro 1}

\section{Estrategias didácticas desarrolladas y evaluadas en el curso "Fundamentos de las Ciencias Bibliotecológicas y de la}

\section{Información"}

\begin{tabular}{|c|c|c|c|}
\hline Estrategia & Objetivo & Descripción & Aspectos evaluados \\
\hline $\begin{array}{l}\text { Un taller sobre } \\
\text { construcción de } \\
\text { ecobotellas }\end{array}$ & $\begin{array}{l}\text { Reconocer la labor del } \\
\text { profesional en bibliotecología } \\
\text { y su aporte a los objetivos de } \\
\text { Desarrollo Sostenible de } \\
\text { Naciones Unidas. }\end{array}$ & $\begin{array}{l}\text { Se ofreció una charla sobre gestión integral } \\
\text { de residuos y posteriormente se desarrolló un } \\
\text { taller donde los estudiantes elaboraron de } \\
\text { forma individual una ecobotella. } \\
\text { Adicionalmente investigaron sobre iniciativas } \\
\text { de otras bibliotecas en el marco de la Agenda } \\
2030 \text { y cada uno propuso una estrategia para } \\
\text { utilizar ecobotellas en una biblioteca o en su } \\
\text { comunidad, esto es parte de una de las } \\
\text { unidades temáticas del curso sobre el rol } \\
\text { social del profesional en bibliotecología. }\end{array}$ & $\begin{array}{l}\text { Preguntas cerradas: } \\
\text {-Grado de dificultad para conseguir los } \\
\text { materiales del taller. } \\
\text {-Conocimiento sobre los temas tratados } \\
\text { y utilidad para la vida. } \\
\text {-Opinión sobre la organización del } \\
\text { taller. } \\
\text { Preguntas abiertas: } \\
\text {-Si la actividad despertó el interés. } \\
\text {-Sugerencias adicionales para la } \\
\text { actividad. }\end{array}$ \\
\hline $\begin{array}{lr}\text { Una } & \text { gira } \\
\text { educativa } & \text { al } \\
\text { Instituto } & \\
\text { Tecnológico de } \\
\text { Costa Rica }\end{array}$ & $\begin{array}{l}\text { Analizar las características } \\
\text { de las bibliotecas en la } \\
\text { actualidad desde la realidad } \\
\text { de una biblioteca } \\
\text { universitaria tradicional y un } \\
\text { Learning Commons. }\end{array}$ & $\begin{array}{l}\text { Se llevó a cabo una visita guiada a } 2 \\
\text { unidades de información: una biblioteca } \\
\text { universitaria y un Learning Commons. Los } \\
\text { estudiantes tuvieron la oportunidad de } \\
\text { conocer la particularidad de cada unidad e } \\
\text { intercambiar conocimiento con los } \\
\text { profesionales a cargo. }\end{array}$ & $\begin{array}{l}\text { Preguntas cerradas: } \\
\text {-Se consultó sobre el grado de } \\
\text { conocimiento de la labor del profesional } \\
\text { en bibliotecología. } \\
\text {-Si el estudiante conocía acerca de los } \\
\text { Learning Commons. } \\
\text {-Si la gira estuvo bien organizada y si le } \\
\text { agradó la actividad. }\end{array}$ \\
\hline
\end{tabular}




\begin{tabular}{|c|c|c|c|}
\hline & & & $\begin{array}{l}\text { Preguntas abiertas: } \\
\text { - ¿Cómo considera que debe ser el rol } \\
\text { actual del profesional en } \\
\text { bibliotecología? } \\
\text { - ¿Tiene algún otro comentario o } \\
\text { sugerencia? }\end{array}$ \\
\hline $\begin{array}{l}\text { Una clase } \\
\text { teórica } \\
\text { acompañada de } \\
\text { un trabajo } \\
\text { práctico manual } \\
\text { en equipo: } \\
\text { Elaboración de } \\
\text { libro botón }\end{array}$ & $\begin{array}{l}\text { Comprender la historia de la } \\
\text { escritura, el libro y las } \\
\text { bibliotecas }\end{array}$ & $\begin{array}{l}\text { Se impartió una clase teórica donde las } \\
\text { docentes a cargo explicaron la historia de la } \\
\text { escritura, el libro y las bibliotecas, además se } \\
\text { culminó esta clase con una demostración } \\
\text { sobre elaboración de audio libros y se les } \\
\text { asignó leer un cuento sobre esta temática: } \\
\text { "Autobiografía del libro" de Virginia de } \\
\text { Fonseca. La semana siguiente los estudiantes } \\
\text { elaboraron un libror botón } \\
\text { (http://lapiceromagico.blogspot.com/2014/03/li } \\
\text { bro-boton.html), un recurso didáctico manual } \\
\text { de construcción conjunta en un ambiente } \\
\text { colaborativo, con el cual recordaron y } \\
\text { sintetizaron los contenidos de la clase teórica. } \\
\text { Este recurso fue presentado a los demás } \\
\text { compañeros para su discusión. }\end{array}$ & $\begin{array}{l}\text { Preguntas cerradas: } \\
\text {-Grado de dificultad para conseguir los } \\
\text { materiales del taller. } \\
\text {-Si la actividad le ayudó a recordar sus } \\
\text { conocimientos sobre historia de la } \\
\text { escritura, del libro y las bibliotecas. } \\
\text {-Si considera que la actividad le ayudó } \\
\text { a desarrollar su capacidad de síntesis. } \\
\text {-Si le gusta que se realice esta } \\
\text { actividad en el curso. } \\
\text { Preguntas abiertas: } \\
\text { - ¿Considera que este tipo de actividad } \\
\text { le ayuda a trabajar de manera } \\
\text { colaborativa? } \\
\text { - ¿Tiene algún otro comentario o } \\
\text { sugerencia? }\end{array}$ \\
\hline
\end{tabular}

Fuente: elaboración propia de las personas autoras, 2021. 
Bibliotecas. Vol. 40, № 1, enero - junio, 2022. EISSN: 1659-3286

URL: http://www.revistas.una.ac.cr/index.php/bibliotecas/index

\section{Resultados}

a. Diagnóstico sobre la situación de aprendizaje y características de los estudiantes

Cuadro 2

Características generales de los estudiantes del curso Fundamentos de las Ciencias Bibliotecológicas y de la información, UCR 2019

\begin{tabular}{|c|c|c|c|}
\hline \multicolumn{3}{|c|}{ Característica } & Cantidad \\
\hline \multirow{2}{*}{\multicolumn{3}{|c|}{$\begin{array}{l}\text { Total de estudiantes } \\
\text { Edad mediana(Ranao 17-59) }\end{array}$}} & 56 \\
\hline & \multicolumn{2}{|c|}{ Edad mediana(Rango:17-59) } & 19 años \\
\hline \multirow{2}{*}{ Sexo } & \multicolumn{2}{|r|}{ Hombres } & 17 \\
\hline & \multicolumn{2}{|r|}{ Mujeres } & 39 \\
\hline \multirow{2}{*}{ Énfasis } & \multicolumn{2}{|c|}{ Bibliotecas educativas } & 30 \\
\hline & \multicolumn{2}{|c|}{ Ciencias de la información } & 26 \\
\hline \multirow{2}{*}{\multicolumn{2}{|c|}{ Estudiante de primer ingreso }} & Sí & 49 \\
\hline & & No & 7 \\
\hline \multirow{2}{*}{\multicolumn{2}{|c|}{ Recibe beca }} & Sí & 40 \\
\hline & & No & 16 \\
\hline \multirow{2}{*}{\multicolumn{2}{|c|}{ Esta es la carrera que desea cursar }} & Sí & 33 \\
\hline & & No & 23 \\
\hline
\end{tabular}

Fuente: elaboración propia de las personas autoras, 2021.

De la información anterior, es importante destacar que en este curso predominan los adultos jóvenes que están iniciando sus estudios en la Universidad de Costa Rica y una parte de ellos no desean cursar la carrera, lo cual sugiere la necesidad de implementar estrategias didácticas innovadoras y dinámicas que despierten el interés por la materia desarrollada en clase.

La mayoría presenta una condición económica limitada, lo que implicó que a la hora de desarrollar estrategias didácticas y de evaluación se solicitaran materiales de bajo costo y el apoyo de la Universidad para cubrir el transporte a la gira, reduciendo así el aporte económico del grupo. Los sujetos de investigación en su mayoría están inscritos en el Bachillerato de Bibliotecas Educativas, por lo cual se procuró proponer estrategias adecuadas al perfil de 
Bibliotecas. Vol. 40, № 1, enero - junio, 2022. EISSN: 1659-3286

URL: http://www.revistas.una.ac.cr/index.php/bibliotecas/index

salida solicitado por este Bachillerato. En cuanto a elementos que podrían afectar en el hecho pedagógico de los estudiantes, se consultó sobre diversos aspectos, categorizados en: aspectos del entorno, actividades en clase y trabajo personal.

En el Gráfico 1, se muestran los aspectos relacionados con el entorno, el más relevante que afecta el hecho pedagógico es la necesidad de tener un receso durante el período de clase para aprender mejor.

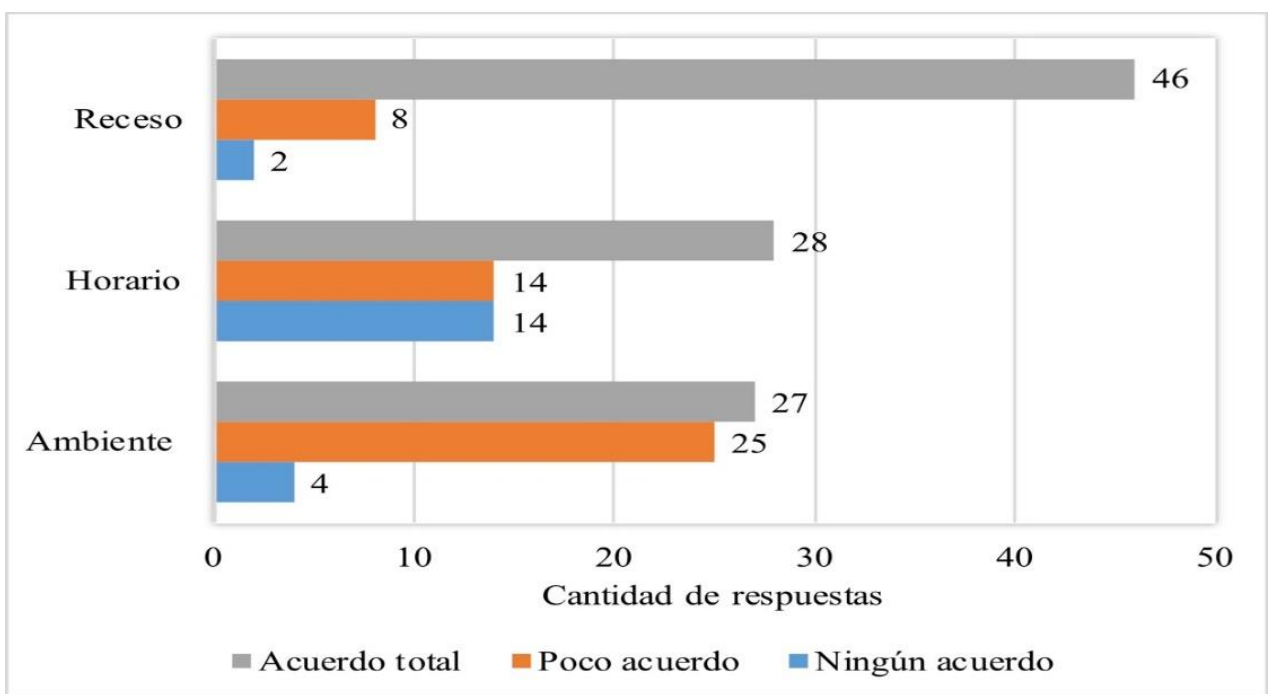

Gráfico 1. Aspectos del entorno que podrían afectar en el hecho pedagógico, según opinión de los estudiantes del curso Fundamentos de las Ciencias Bibliotecológicas y de lainformación, UCR 2019 (en valores absolutos)

Fuente: elaboración propia de las personas autoras, 2021.

En cuanto a las actividades en clase, pareciera que este tipo de población se inclina por las kinestésicas, ya que prefieren actividades recreativas, giras, uso de la tecnología, aprender haciendo y también manifiestan necesitar las instrucciones de lo que deben realizar por escrito (ver Gráfico 2). En la dimensión de trabajo personal los estudiantes manifiestan que es muy importante la relación que tengan con el docente. 
Bibliotecas. Vol. 40, № 1, enero - junio, 2022. EISSN: 1659-3286

URL: http://www.revistas.una.ac.cr/index.php/bibliotecas/index

También expresan que, para apoyar su proceso de aprendizaje, les es útil hacer tareas y en general prefieren el trabajo individual (Gráfico 3).Esta información arrojó la necesidad de desarrollar algunas sesiones fuera del espacio habitual, visitar lugares distintos, alternar trabajos colectivos e individuales, acompañar la teoría con actividades prácticas, tener sesiones de discusión e intercambio de opiniones, la importancia de la implementación de rúbricas de evaluación con instrucciones claras, además, la relevancia de utilizar el aula virtual institucional como apoyo didáctico, propiciar una relación armónica estudiantes-docente y, por último, respetar el tiempo de receso establecido para el periodo de clase.



Gráfico 2. Aspectos relacionados con las actividades en clase que podrían afectar en el hecho pedagógico, según opinión de los estudiantes del curso Fundamentos de las Ciencias Bibliotecológicas y de la información, UCR 2019 (en valores absolutos)

Fuente: elaboración propia de las personas autoras, 2021. 
Bibliotecas. Vol. 40, № 1, enero - junio, 2022. EISSN: 1659-3286

URL: http://www.revistas.una.ac.cr/index.php/bibliotecas/index



Gráfico 3. Aspectos relacionados con el trabajo personal que podrían afectar en el hecho pedagógico, según opinión de los estudiantes del curso Fundamentos de las Ciencias Bibliotecológicas y de la información, UCR 2019 (en valores absolutos)

Fuente: elaboración propia de las personas autoras, 2021.

De acuerdo con el análisis realizado sobre el trabajo extracurricular y responsabilidad del estudiante, se descubrió que el $87,5 \%$ son conscientes de la importancia de realizar aquellas actividades que forman parte de la evaluación (tareas y prácticas), sin embargo, solamente $23,2 \%$ de los estudiantes se consideran totalmente autodidactas y el $32,1 \%$ afirman estudiar por su cuenta si no asisten a la clase. Esta realidad demuestra la importancia de recibir clases presenciales y con actividades evaluadas.

\section{b. Mejor manera de aprender}

Esta fue una pregunta abierta, por lo tanto, las autoras analizaron cada una de las respuestas de los estudiantes y definieron categorías que se presentan a continuación, se destaca la importancia de llevar a la práctica lo aprendido:

- Actividades practices.

- Discusiones en clase.

- Actividades que incentiven la creatividad. 
Bibliotecas. Vol. 40, № 1, enero - junio, 2022. EISSN: 1659-3286

URL: http://www.revistas.una.ac.cr/index.php/bibliotecas/index

- La respuesta no se relacionaba con la pregunta.

- Repaso de la teoría.

- Uso de imágenes para asociar el aprendizaje a la vida cotidiana.

- Uso de tecnología.

\section{c. Estrategias didácticas recomendadas para el profesor}

Esta fue una pregunta abierta y las autoras definieron categorías que describen las respuestas de los estudiantes. Se presentan más de 56 respuestas pues en ocasiones los estudiantes escribieron varias opciones:

- Prácticas en clase.

- Giras académicas.

- Uso de juegos y actividades creativas.

- Uso de herramientas tecnológicas.

- La respuesta no se relacionaba con la pregunta o no respondió.

- Debates de opinión.

- Lecturas académicas.

- Uso de presentaciones.

\section{d. Fortalezas con que cuentan los estudiantes}

También se les consultó a los estudiantes, con qué fortalezas contaban para estudiar y aprender, aspectos relacionados con la asistencia y el trabajo dentro del aula fueron los más prevalentes (Cuadro 3). También manifestaron tener acceso a recursos de información como una fortaleza. 
Bibliotecas. Vol. 40, $\mathrm{N}^{\circ} 1$, enero - junio, 2022. EISSN: 1659-3286

URL: http://www.revistas.una.ac.cr/index.php/bibliotecas/index

\section{Cuadro 3}

Fortalezas de los estudiantes del curso Fundamentos de las Ciencias Bibliotecológicas y de la información identifican, UCR 2019

\begin{tabular}{|l|c|}
\hline \multicolumn{1}{|c|}{ Fortaleza } & Estudiantes que la tienen \\
\hline Atención a clases & 49 \\
\hline Asistencia constante & 47 \\
\hline Tomar apuntes & 45 \\
\hline Hacer tareas & 42 \\
\hline Hacer prácticas & 41 \\
\hline Uso de tecnologías y material de apoyo & 41 \\
\hline Orden y disciplina & 38 \\
\hline Motivación y alegría & 36 \\
\hline Dinamismo y creatividad & 35 \\
\hline Apoyo familiar & 31 \\
\hline Estudiar la materia fuera de clase & 30 \\
\hline Amor a la carrera & 30 \\
\hline Formular preguntas durante y después de clase & 21 \\
\hline Búsqueda de material extra & 20 \\
\hline
\end{tabular}

Fuente: elaboración propia de las personas autoras, 2021.

\section{e. Actividades que facilitan el aprendizaje de los estudiantes}

De la información del Cuadro 4 y para planear y desarrollar las estrategias didácticas que se propusieron, se tomó muy en cuenta que los estudiantes, aparte de la teoría que ya se imparte en el aula, necesitaban complementar el aprendizaje de esta por medio de actividades prácticas y creativas. También se consideró generar un balance entre el trabajo individual y colaborativo, ya que el perfil de salida de las carreras así lo amerita. 
Bibliotecas. Vol. 40, № 1, enero - junio, 2022. EISSN: 1659-3286

URL: http://www.revistas.una.ac.cr/index.php/bibliotecas/index

Es importante destacar, además, que los estudiantes indicaron que aprenden de mejor manera realizando discusiones, tareas y a través de experiencias prácticas dentro y fuera del aula de forma presencial. Es por esta razón que las tres estrategias didácticas desarrolladas para efectos de esta investigación buscaron la diversidad e ingenio, la aplicabilidad de la teoría vista en clase por medio de ejemplos vivenciales que, además, fueron parte de los rubros evaluados en el curso, por lo tanto, las investigadoras pudieron medir también el aprendizaje obtenido por los sujetos de investigación.

\section{Cuadro 4}

Actividades que facilitan el aprendizaje de los estudiantes del curso Fundamentos de las Ciencias Bibliotecológicas y de la información identifican, UCR 2019

\begin{tabular}{|l|c|}
\hline \multicolumn{1}{|c|}{ Actividad } & Estudiantes \\
\hline Giras y trabajo de campo & 51 \\
\hline Las discusiones y análisis de casos concretos en clase & 47 \\
\hline Prácticas & 41 \\
\hline Presentaciones con diapositivas & 34 \\
\hline Aula virtual & 30 \\
\hline Uso de videos & 29 \\
\hline Quiz & 26 \\
\hline Investigaciones de los cursos & 26 \\
\hline Lecturas & 26 \\
\hline Mesas redondas & 20 \\
\hline
\end{tabular}

Fuente: elaboración propia de las personas autoras, 2021.

\section{f. Resultados de la estrategia didáctica "Taller sobre construcción de ecobotellas"}

Esta evaluación fue realizada por 54 estudiantes. A continuación, se presentan sus valoraciones en cuanto a los cuestionamientos planteados. En el Gráfico 4 se puede observar que la actividad fue bien asimilada ya que a la mayoría le gustó la estrategia, la consideraron 
Bibliotecas. Vol. 40, № 1, enero - junio, 2022. EISSN: 1659-3286

URL: http://www.revistas.una.ac.cr/index.php/bibliotecas/index

bien organizada, manifestaron que aumentó el conocimiento aprendido y es útil para la vida. Cabe recalcar que el obtener los materiales para realizar ecobotellas es relativamente fácil.

En cuanto a la pregunta ¿Considera que la actividad despertó su interés? Los estudiantes respondieron que sí, además 29 de ellos indicaron que "se creó conciencia ambiental" y 25 comentaron que "se generó conocimiento nuevo y aplicable para la vida". Respecto a la consulta ¿Tiene algún otro comentario o sugerencia? Esta fue respondida por 41 de los estudiantes: 19 comentaron que tienen interés en continuar prácticas ambientales amigables, 10 de ellos dieron sugerencias para mejorar la actividad, 9 expresaron que es una actividad que dinamiza el curso y 3 indicaron que es una actividad de interés profesional.

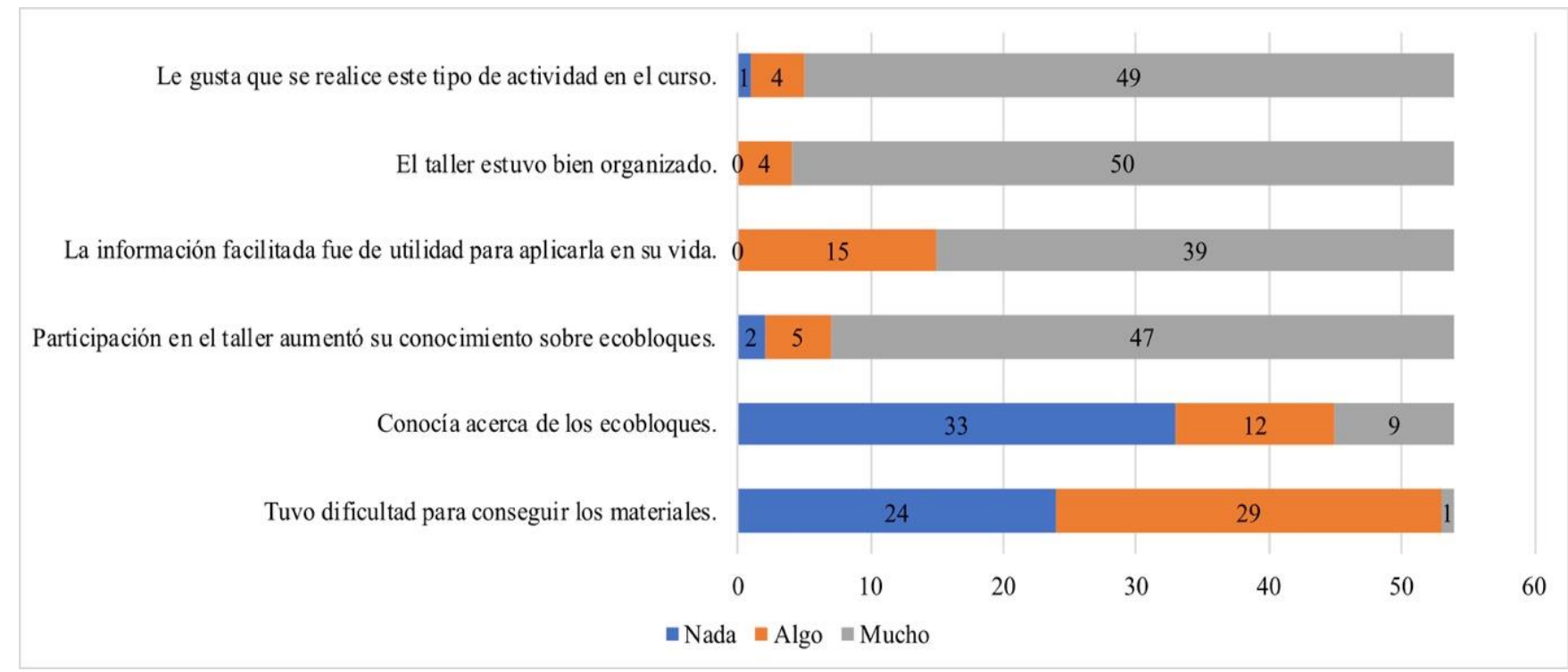

Gráfico 4. Diferentes aspectos relacionados con la evaluación del taller para crear ecobotellas, según opinión de los estudiantes del curso Fundamentos de las Ciencias Bibliotecológicas y de la información, UCR 2019 (en valores absolutos)

Fuente: elaboración propia de las personas autoras, 2021. 
Bibliotecas. Vol. 40, № 1, enero - junio, 2022. EISSN: 1659-3286

URL: http://www.revistas.una.ac.cr/index.php/bibliotecas/index

\section{g. Resultados de la estrategia didáctica "Gira educativa al Instituto Tecnológico de Costa Rica"}

Esta evaluación fue realizada por 53 estudiantes. De acuerdo con lo manifestado en el diagnóstico, la actividad tuvo mucha acogida, pues la mayoría respondió que les gustó "mucho" (Gráfico 5), además reconocieron que estuvo bien organizada. Se considera que la gira aportó a la generación del conocimiento debido a que los estudiantes manifestaron que no conocían o conocían poco sobre los Learning Common.

Respecto a la pregunta ¿Cómo considera usted que debe ser el rol actual del bibliotecólogo?, la mayoría (43 estudiantes) considera que debe ser un profesional activo y actualizado en el manejo de información. Algunos mencionaron ser profesionales formadores, creativos y preservadores de la información (Gráfico 6).

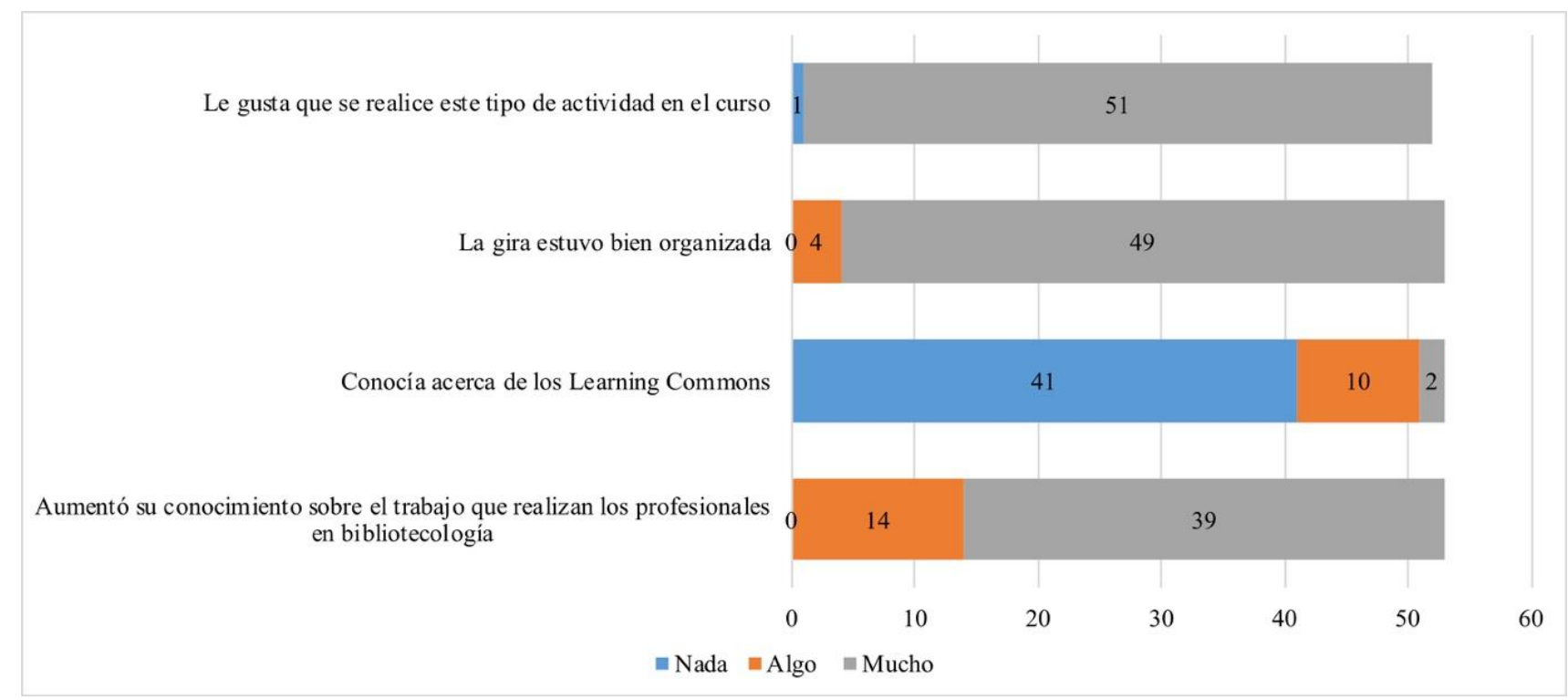

Gráfico 5. Diferentes aspectos relacionados con la evaluación de la gira a un Common Learning, según opinión de los estudiantes del curso Fundamentos de las Ciencias Bibliotecológicas y de la información, UCR 2019 (en valores absolutos)

Fuente: elaboración propia de las personas autoras, 2021. 
Bibliotecas. Vol. 40, № 1, enero - junio, 2022. EISSN: 1659-3286

URL: http://www.revistas.una.ac.cr/index.php/bibliotecas/index

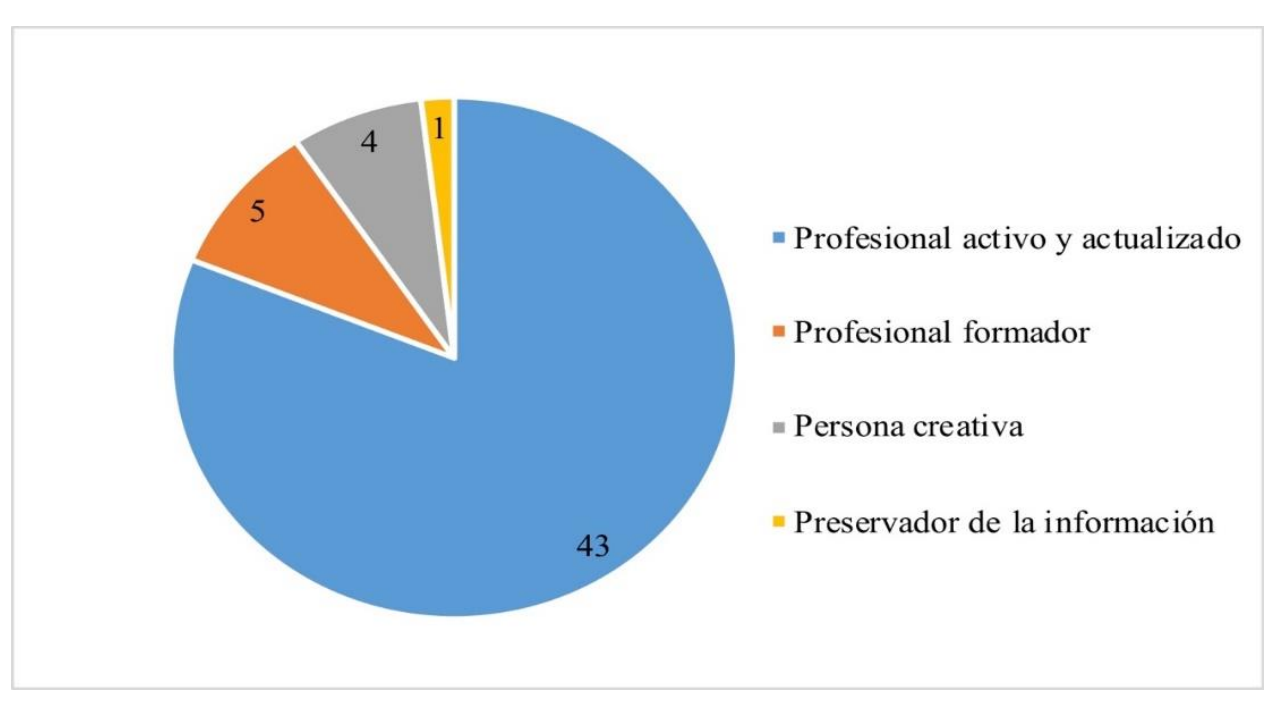

Gráfico 6: Diferentes aspectos relacionados con el rol actual del profesional en bibliotecología, según opinión de los estudiantes del curso Fundamentos de las Ciencias Bibliotecológicas y de la información, UCR 2019 (en valores absolutos)

Fuente: elaboración propia de las personas autoras, 2021.

En cuanto a la pregunta, ¿Tiene algún otro comentario o sugerencia? Fue respondida por 28 estudiantes: 10 indicaron que la actividad favorece el aprendizaje, 9 sugirieron continuar con este tipo de actividad, 5 expresaron que aumentó su interés por el curso y 4 dieron sugerencias para mejorar la gira.

\section{h. Resultados de la estrategia didáctica "Taller de elaboración de libro botón"}

Esta evaluación fue realizada por 43 estudiantes. La mayoría manifestó que la actividad fue muy provechosa debido a que les gustó y les ayudó a desarrollar la capacidad de síntesis, recordar la historia del libro, la escritura y las bibliotecas (gráfico 7). 
Bibliotecas. Vol. 40, № 1, enero - junio, 2022. EISSN: 1659-3286

URL: http://www.revistas.una.ac.cr/index.php/bibliotecas/index

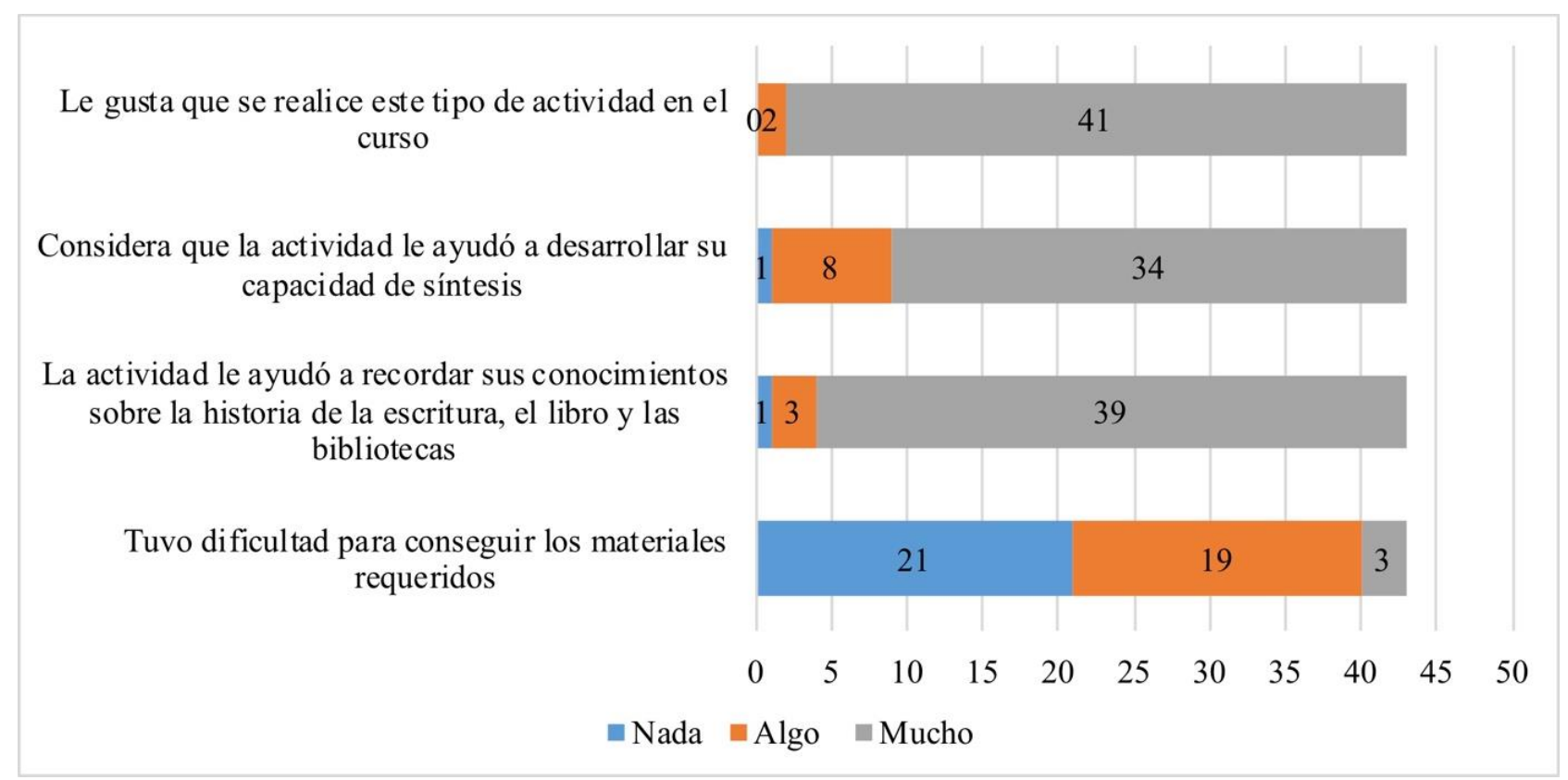

Gráfico 7. Diferentes aspectos relacionados con la evaluación de la construcción del libro botón, según opinión de los estudiantes del curso Fundamentos de las Ciencias Bibliotecológicas y de la información, UCR 2019(en valores absolutos) Fuente: elaboración propia de las personas autoras, 2021.

Respecto a la pregunta ¿Considera que este tipo de actividad le ayuda a trabajar de manera colaborativa? En el gráfico 8 se muestran las opiniones de los estudiantes; cerca de la mitad considera que la actividad del libro botón favorece la comunicación y el aprendizaje colaborativo, principalmente. 
Bibliotecas. Vol. 40, № 1, enero - junio, 2022. EISSN: 1659-3286

URL: http://www.revistas.una.ac.cr/index.php/bibliotecas/index

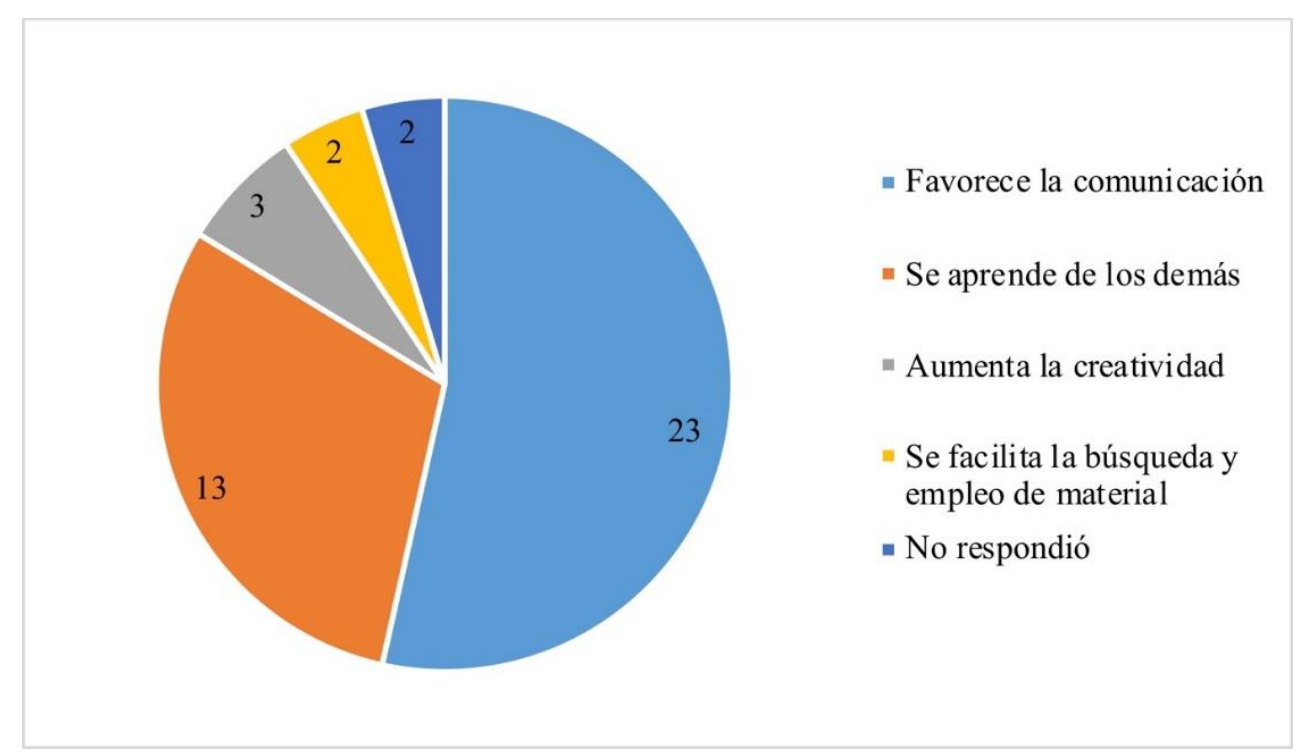

Gráfico 8. Distribución de los estudiantes del curso "Fundamentos de las Ciencias Bibliotecológicas y de la información", UCR 2019, según opinión sobre trabajo colaborativo. Fuente: elaboración propia de las personas autoras, 2021.

En cuanto a la pregunta ¿Tiene algún otro comentario o sugerencia? Fue respondida por 25 estudiantes: 9 indicaron que la estrategia aumento su interés por la materia, 7 sugirieron continuar con este tipo de actividad, 5 comentaron que se favoreció el aprendizaje, 2 recomendaron ampliar el tiempo asignado para la actividad y 2 sugirieron reconsiderar el tipo de materiales usados.

\section{Conclusiones}

A raíz de la experiencia educativa vivida durante el I ciclo 2019 en el curso Bl-1002 Fundamentos de las Ciencias Bibliotecológicas y de la Información se considera importante combinar las prácticas educativas tradicionales con estrategias didácticas creativas, dinámicas, prácticas e ingeniosas, donde el estudiantado, además, pueda investigar y aportar opiniones críticas que contribuyan con el trabajo del docente en el aula, favoreciendo un rol más activo por parte del estudiante, donde se manifiesta una construcción de trabajo colectivo y con un enfoque dialógico por ambas partes. 
Bibliotecas. Vol. 40, № 1, enero - junio, 2022. EISSN: 1659-3286

URL: http://www.revistas.una.ac.cr/index.php/bibliotecas/index

Para las investigadoras, estas fueron estrategias didácticas significativas que permitieron alcanzar los objetivos buscados con la aplicación de cada una de ellas. Los estudiantes obtuvieron buenas calificaciones en cuanto a los rubros evaluados, además, fue posible desarrollar habilidades blandas como la comunicación asertiva, el trabajo colaborativo, la observación, la percepción y la capacidad de análisis. Asimismo, se fortalecieron diferentes ejes transversales relacionados con las carreras, especialmente el relacionado con la educación ambiental.

Es importante destacar que la aplicación de estrategias didácticas innovadoras conlleva para el docente un trabajo y planificación más robusto y demandante, sin embargo, los resultados obtenidos son más relevantes y eficaces en cuanto a conocimientos, motivaciones y espacios de socialización para los estudiantes, resaltando la importancia de considerar estos no como algo pasajero, sino como una experiencia integral.

Las estrategias didácticas puestas en práctica favorecieron la dinámica del curso, ya que en una evaluación docente realizada por el Centro de Evaluación Académica de la Universidad de Costa Rica (CEA), las investigadoras obtuvieron una calificación de 9,75 de 10 en este curso. Esta evaluación es realizada por los mismos estudiantes y califica el trabajo del docente en el aula y su pertinencia para el aprendizaje de la materia del curso.

En cuanto al trabajo con grupos grandes se presentan distintos retos, entre ellos aplicar estrategias didácticas innovadoras que realmente impacten en el grupo, ya que en espacios de aprendizaje numerosos la interacción por parte del docente se hace más compleja por la atención que los estudiantes demandan, además, influyen los horarios, los recursos materiales disponibles y las características del espacio físico. Se recalca que los métodos de evaluación del aprendizaje requieren una adaptación donde se combinen evaluaciones individuales y colectivas, ya que se debe considerar el tiempo de aplicación de las pruebas y, también, la revisión por parte del docente. 
Bibliotecas. Vol. 40, № 1, enero - junio, 2022. EISSN: 1659-3286

URL: http://www.revistas.una.ac.cr/index.php/bibliotecas/index

Para finalizar, las autoras consideran que las estrategias didácticas innovadoras, bien planificadas y adaptadas a las necesidades de los estudiantes producen conocimiento, motivación y curiosidad para continuar descubriendo los aprendizajes de las carreras de bibliotecología, en miras a formar profesionales capacitados a nivel técnico y social.

\section{Referencias:}

Araya, A. (2016). La dimensión lúdica del ser humano en el aprendizaje. San José, CR: EUNED.

Escuela de Bibliotecología y Ciencias de la Información [EBCI] (2019). BI-1001 Fundamentos de las Ciencias Bibliotecológicas y de la Información: Programa del curso. San José, Costa Rica: EBCI.

González, B. y León, A. (2009). Interacción verbal y socialización cognitiva en el aula de clase. Acción Pedagógica, 18, 30-41. Recuperado de https://dialnet.unirioja.es/servlet/articulo?codigo $=3122358$

González, L. (2013). Learning commons en bibliotecas académicas. Biblios: Revista de Bibliotecología y Ciencias de la Información, 53, 88-96. DOI: https://doi.org/10.5195/biblios.2013.136

Hernández, R., Fernández, C. y Baptista, M. (2010). Metodología de la investigación (5 ed.). México: McGrawHill

Imbernón, F. y Medina, J.L. (2006). Metodología participativa en el aula universitaria: La participación del alumnado. En Martínez, M. (coord.), Propuestas para el cambio docente en la universidad (91-122). España: Editorial Octaedro.

Muñoz Rocha, C.I. (2015). Metodología de la investigación. México: Oxford University Press

Piedra García, L.A. (2015). Una aproximación científica y pedagógica a la existencia de los grupos grandes en el contexto universitario. San José, Costa Rica: DEDUN

Piedra, L.A. y Arias, M. (2015). Informe final del proyecto: Creencias y representaciones del estudiantado en los procesos de formación de la Universidad de Costa Rica. Recuperado de http://repositorio.inie.ucr.ac.cr/bitstream/123456789/375/1/06.03.06\%202280.pdf 
Bibliotecas. Vol. 40, № 1, enero - junio, 2022. EISSN: 1659-3286

URL: http://www.revistas.una.ac.cr/index.php/bibliotecas/index

Programa Estado de la Educación (2019). Estado de la Educación Costarricense 2019. Recuperado de http://repositorio.conare.ac.cr/handle/20.500.12337/7773

Sobrados, M. (2016). El trabajo docente en grupos numerosos: Experiencias en el uso del portafolio. Opción, 10, 773-788. Recuperado de https://www.redalyc.org/pdf/310/31048901043.pdf

\section{Notas de las personas autoras:}

Lorena Chaves Salgado: académica de la Escuela de Bibliotecología y Ciencias de la Información, Universidad de Costa Rica. Correo electrónico: lorena.chaves@ucr.ac.cr. ORCID ID: https://orcid.org/0000-0001-9169-7541

Daniela Rodríguez Valerio: académica de la Escuela de Bibliotecología y Ciencias de la Información, Universidad de Costa Rica. Correo electrónico: daniela.rodriguezvalerio@ucr.ac.cr. ORCID ID: https://orcid.org/0000-0003-3737-8851

Ericka Méndez Chacón: Académica de la Universidad de Costa Rica; labora en Escuela de Bibliotecología y Ciencias de la Información, la Escuela de Estadística y en el Centro Centroamericano de Población. Correo electrónico: ericka.mendez@ucr.ac.cr. ORCID ID: https://orcid.org/0000-0003-3152-5179 